\title{
A Force Limiting Adaptive Controller for a Robotic System Undergoing a Noncontact-to-Contact Transition
}

\author{
Chien-Hao Liang, Student Member, IEEE, Shubhendu Bhasin, Student Member, IEEE, \\ Keith Dupree, Student Member, IEEE, and Warren E. Dixon, Senior Member, IEEE
}

\begin{abstract}
The problem of prescribing, reducing, or controlling the interaction forces between a robot and the environment during a noncontact-to-contact transition is intriguing because large interaction forces can damage both the robot and/or the environment or lead to degraded performance or instabilities. In this paper, we consider a two-link planar robotic arm that transitions from free motion to contact with an unactuated mass-spring system. The objective is to control a robot from a noncontact initial condition to a desired (in-contact) position so that the mass-spring system is regulated to a desired compressed state. The feedback elements for the controller in this paper are contained inside hyperbolic tangent functions as a means to limit the impact forces resulting from large initial conditions as the robot transitions from a noncontact to contact state. The main challenge of this work is that the use of saturated feedback does not allow some coupling terms to be canceled in the stability analysis, resulting in the need to develop state-dependent upper bounds that reduce the stability to a semiglobal result. New control development, closed-loop error systems, and Lyapunov-based stability analysis arguments are used to conclude the result. It is interesting to note that only the position and velocity terms are required for the proposed controller (i.e., the controller does not depend on measuring the impact force and the acceleration terms). Experimental results that successfully demonstrate the control objective are provided.
\end{abstract}

Index Terms-Actuator constraint, adaptive control, Lyapunov method.

\section{INTRODUCTION}

$\mathbf{T}$ HE PROBLEM of controlling a robot during a noncontact-to-contact transition has been a historically challenging problem that is practically motivated by applications that require a robotic system to interact with the environment. The control challenge is due, in part, to the impact effects that result in possible high stress, rapid dissipation of energy, and

Manuscript received June 05, 2007; revised April 16, 2008. Manuscript received in final form September 29, 2008. First published April 14, 2009; current version published October 23, 2009. Recommended by Associate Editor N. Hovakimyan. This work was supported in part by NSF under CAREER Award 0547448, by AFOSR under Contract F49620-03-1-0170, and by the Department of Energy under Grant DE-FG04-86NE37967 as part of the DOE University Research Program in Robotics.

C.-H. Liang, S. Bhasin, and W. E. Dixon are with the Department of Mechanical and Aerospace Engineering, University of Florida, Gainesville, FL 32611 USA (e-mail: chliang@ufl.edu; sbhasin@ufl.edu; wdixon@ufl.edu).

K. Dupree is with the Nonlinear Controls and Robotics Group, Department of Mechanical and Aerospace Engineering and the Department of Electrical and Computer Engineering, University of Florida, Gainesville, FL 32611 USA (e-mail: kdupree@ufl.edu).

Color versions of one or more of the figures in this paper are available online at http://ieeexplore.ieee.org.

Digital Object Identifier 10.1109/TCST.2008.2007573 fast acceleration and deceleration as stated in [1]. Over the last two decades, results such as [2]-[44] have focused on control designs that can be applied during the noncontact-to-contact transition. One main theme in these results is the desire to prescribe, reduce, or control the interaction forces during or after the robot impact with the environment such as [2]-[25] because large interaction forces can damage both the robot and/or the environment or lead to degraded performance or instabilities.

Among [2]-[25], two main approaches have been exploited to accommodate for the noncontact-to-contact transition. The first approach is to exploit the kinematic redundancy of the manipulator to reduce the impact force [4]-[6]. The second mainstream approach is to exploit a discontinuous controller that switches based on the different phases of the dynamics (i.e., noncontact, robot impact transition, and in-contact coupled manipulator and environment) as in [7]-[18]. Typically, these discontinuous controllers consist of a velocity controller in the precontact phase that switches to a force controller during the in-contact phase. Motivation exists to explore alternative methods because kinematic redundancy is not always possible, and discontinuous controllers require infinite control frequency (i.e., exhibit chattering) or yield degraded stability results (i.e., uniformly ultimately bounded).

In this paper, we consider a two-link planar robotic arm that transitions from free motion to contact with an unactuated mass-spring system. The robot/mass-spring-system collision is modeled as a differentiable impact as in recent work in [1] and [26] and in our previous efforts in [27]-[30]. As in our previous efforts in [27] and [28], the objective is to control a robot from a noncontact initial condition to a desired (in-contact) position so that the mass-spring system is regulated to a desired compressed state. The focus of our previous work was to develop a continuous exact model knowledge [27] and adaptive controller [28] that could achieve the objective despite the impact collision disturbance. When these results were implemented in the presence of large initial conditions, a violent impact between the robot and the mass-spring system resulted. In fact, the controller was artificially saturated (the saturation effects were not considered in the stability analysis) to reduce the impact forces so that the mass deflection would not destroy the capacitance probe. These results provide the motivation for the current control development. Specifically, the feedback elements for the controller in this paper are contained inside hyperbolic tangent functions as a means to limit the impact forces resulting from large initial conditions as the robot transitions from noncontact 


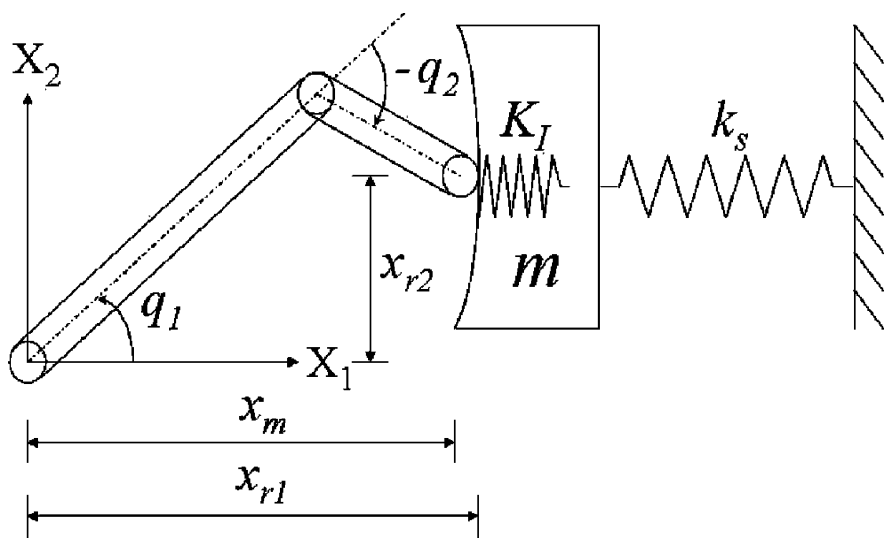

Fig. 1. MSR system is an academic example of an impact between two dynamic systems.

to contact. Although saturating the feedback error is an intuitive solution that has been proposed in previous literature for other types of robotic systems with limited actuation (e.g., see [45] and the references within), several new technical challenges arise due to the impact condition. The main challenge is that the use of saturated feedback does not allow some coupling terms to be canceled in the stability analysis, resulting in the need to develop state-dependent upper bounds that reduce the stability to a semiglobal result (as compared to the global results in [27]-[30]). The semiglobal result is problematic in the current applicative context because certain control terms do not appear in the closed-loop error system during the noncontact condition, resulting in a uniformly ultimately bounded result until the robot makes contact. Hence, the result hinges on new development within the semiglobal stability proof for an error system that is only uniformly ultimately bounded during the noncontact phase. This problem is exacerbated by the fact that the Lyapunov function contains radially unbounded hyperbolic functions of some states that only appear inside saturated hyperbolic terms in the Lyapunov derivative. New control development, closed-loop error systems, and Lyapunov-based stability-analysis arguments are used to conclude the result. It is interesting to note that only the position and velocity terms of the mass-spring system and the joint angles and the angular velocity terms of the planar robotic arm are required for the proposed controller (i.e., the controller does not depend on measuring the impact force and the acceleration terms). Experimental results that successfully demonstrate the control objective are provided.

\section{DYNAMIC MODEL}

The subsequent development is motivated by the academic problem shown in Fig. 1. The dynamic model for the two-link planar robot shown in Fig. 1 can be expressed in the joint space as

$$
M(q) \ddot{q}+C(q, \dot{q}) \dot{q}+h(q)=\tau
$$

where $q(t), \dot{q}(t)$, and $\ddot{q}(t) \in \mathbb{R}^{2}$ represent the angular position, velocity, and acceleration of the robot links, respectively, $M(q) \in \mathbb{R}^{2 \times 2}$ represents the uncertain inertia matrix, $C(q, \dot{q}) \in \mathbb{R}^{2 \times 2}$ represents the uncertain centripetal Coriolis effects, $h(q) \triangleq\left[h_{1}(q), h_{2}(q)\right]^{\mathrm{T}} \in \mathbb{R}^{2}$ represents uncertain conservative forces (e.g., gravity), and $\tau(t) \in \mathbb{R}^{2}$ represents the torque control inputs. The Euclidean position of the end point of the second robot link is denoted by $x_{r}(t) \triangleq\left[x_{r 1}(t), x_{r 2}(t)\right]^{\mathrm{T}} \in \mathbb{R}^{2}$, which can be related to the joint space through the following kinematic relationship:

$$
\dot{x}_{r}=J(q) \dot{q}
$$

where $J(q) \in \mathbb{R}^{2 \times 2}$ denotes the manipulator Jacobian. The unforced dynamics of the mass-spring system in Fig. 1 are

$$
m \ddot{x}_{m}+k_{s}\left(x_{m}-x_{0}\right)=0
$$

where $x_{m}(t), \dot{x}_{m}(t)$, and $\ddot{x}_{m}(t) \in \mathbb{R}$ represent the displacement, velocity, and acceleration of the unknown mass $m \in \mathbb{R}$, $x_{0} \in \mathbb{R}$ represents the initial undisturbed position of the mass, and $k_{s} \in \mathbb{R}$ represents the unknown stiffness of the spring.

Assumption 1: We assume that $x_{r 1}(t)$ and $x_{m}(t)$ can be bounded as

$$
\zeta_{x_{r}} \leq x_{r 1}(t) \quad x_{m}(t) \leq \zeta_{x_{m}}
$$

where $\zeta_{x_{r}} \in \mathbb{R}$ is a known constant that is determined by the minimum coordinate of the robot along the $X_{1}$-axis and $\zeta_{x_{m}} \in$ $\mathbb{R}$ is a known positive constant. The lower bound assumption for $x_{r 1}(t)$ is based on the geometry of the robot, and the upper bound assumption for $x_{m}(t)$ is based on the physical fact that the mass is attached by the spring to some object and the mass will not be able to move past that object.

After premultiplying the robot dynamics by the inverse of the Jacobian transpose and utilizing (2), the dynamics in (1) and (3) can be rewritten as [27], [28]

$$
\begin{aligned}
\bar{M}\left(x_{r}\right) \ddot{x}_{r}+\bar{C}\left(x_{r}, \dot{x}_{r}\right) \dot{x}_{r}+\bar{h}\left(x_{r}\right)+\left[\begin{array}{c}
F_{m} \\
0
\end{array}\right] & =F \\
m \ddot{x}_{m}+k_{s}\left(x_{m}-x_{0}\right) & =F_{m}
\end{aligned}
$$

where $F(t) \triangleq J^{-T}(q) \tau(t) \in \mathbb{R}^{2}$ denotes the manipulator force. In (5) and (6), $F_{m}\left(x_{r}, x_{m}\right) \in \mathbb{R}$ denotes the impact force acting on the mass that occurs when $x_{r 1}(t) \geq x_{m}(t)$ (see Fig. 1) is assumed to have the following form [1], [26]

$$
F_{m}=K_{I} \Lambda\left(x_{r 1}-x_{m}\right)
$$

where $K_{I} \in \mathbb{R}$ represents an unknown positive stiffness constant and $\Lambda\left(x_{r}, x_{m}\right) \in \mathbb{R}$ is defined as

$$
\Lambda= \begin{cases}1 & x_{r 1} \geq x_{m} \\ 0 & x_{r 1}<x_{m}\end{cases}
$$

The dynamic model in (5) exhibits the following properties that will be utilized in the subsequent analysis.

1) Property 1: The inertia matrix $\bar{M}\left(x_{r}\right)$ is symmetric, positive definite, and can be lower and upper bounded as

$$
a_{1}\|\xi\|^{2} \leq \xi^{\mathrm{T}} \bar{M} \xi \leq a_{2}\|\xi\|^{2} \quad \forall \xi \in \mathbb{R}^{2}
$$

where $a_{1}$ and $a_{2} \in \mathbb{R}$ are positive constants. 
2) Property 2: The following skew-symmetric relationship is satisfied:

$$
\xi^{\mathrm{T}}\left(\frac{1}{2} \dot{\bar{M}}\left(x_{r}\right)-\bar{C}\left(x_{r}, \dot{x}_{r}\right)\right) \xi=0 \quad \forall \xi \in \mathbb{R}^{2} .
$$

3) Property 3: The robot dynamics given in (5) can be linearly parameterized as

$$
Y\left(x_{r}, \dot{x}_{r}, \ddot{x}_{r}\right) \theta=\bar{M}\left(x_{r}\right) \ddot{x}_{r}+\bar{C}\left(x_{r}, \dot{x}_{r}\right) \dot{x}_{r}+\bar{h}\left(x_{r}\right)+\left[\begin{array}{c}
F_{m} \\
0
\end{array}\right]
$$

where $\theta \in \mathbb{R}^{p}$ contains the constant unknown system parameters and $Y\left(x_{r}, \dot{x}_{r}, \ddot{x}_{r}\right) \in \mathbb{R}^{2 \times p}$ denotes the known regression matrix.

Assumption 2: We assume that the mass of the mass-spring system can be upper and lower bounded as

$$
m_{l}<m<m_{u}
$$

where $m_{l}$ and $m_{u} \in \mathbb{R}$ denote known positive bounding constants. The unknown stiffness constants $K_{I}$ and $k_{s}$ are also assumed to be bounded as

$$
\underline{\zeta}_{K}<K_{I}<\bar{\zeta}_{K} \quad \underline{\zeta}_{k_{s}}<k_{s}<\bar{\zeta}_{k_{s}}
$$

where $\underline{\zeta}_{K}, \bar{\zeta}_{K}, \underline{\zeta}_{k_{s}}$, and $\bar{\zeta}_{k_{s}} \in \mathbb{R}$ denote known positive bounding constants.

Assumption 3: The subsequent development is based on the assumption that $q(t), \dot{q}(t), x_{m}(t)$, and $\dot{x}_{m}(t)$ are measurable and that $x_{r}(t)$ and $\dot{x}_{r}(t)$ can be obtained from $q(t)$ and $\dot{q}(t)$.

Remark 1: During the subsequent control development, we assume that the minimum singular value of $J(q)$ is greater than a known small positive constant $\delta>0$, such that $\max \left\{\left\|J^{-1}(q)\right\|\right\}$ is known a priori, and hence, all kinematic singularities are always avoided.

Remark 2: To aid the subsequent control design and analysis, we define the vector $\operatorname{Tanh}(\cdot) \in \mathbb{R}^{n}$ as follows:

$$
\operatorname{Tanh}(\delta)=\left[\tanh \left(\delta_{1}\right), \ldots, \tanh \left(\delta_{n}\right)\right]^{\mathrm{T}}
$$

where $\delta=\left[\delta_{1}, \ldots, \delta_{n}\right]^{\mathrm{T}} \in \mathbb{R}^{n}$.

4) Property 4: The following inequalities are valid for all $\xi=\left[\xi_{1}, \ldots, \xi_{n}\right]^{\mathrm{T}} \in \mathbb{R}^{n}[45]:$

$$
\begin{aligned}
\|\xi\| & \geq\|\operatorname{Tanh}(\xi)\| \\
\|\xi\|+1 & \geq \frac{\|\xi\|}{\tanh (\|\xi\|)} \\
\|\xi\|^{2} & \geq \sum_{i=1}^{n} \ln \left(\cosh \left(\xi_{i}\right)\right) \geq \ln (\cosh (\|\xi\|))
\end{aligned}
$$

$\xi^{\mathrm{T}} \operatorname{Tanh}(\xi) \geq \operatorname{Tanh}^{\mathrm{T}}(\xi) \operatorname{Tanh}(\xi)=\|\operatorname{Tanh}(\xi)\|^{2} \geq \tanh ^{2}(\|\xi\|)$.

\section{CONTROL DEVElopment}

The subsequent control design is based on integrator backstepping methods. A desired trajectory is designed for the robot (i.e., a virtual control input) to ensure that the robot converges to and impacts with the mass and to ensure that the robot regulates the mass to the desired position. Since we cannot directly control the robot trajectory, a force controller is developed to ensure that the robot tracks the desired trajectory despite the transition from free motion to an impact collision and despite uncertainties throughout the mass-spring robot (MSR) system.

\section{A. Control Objective}

The control objective is to regulate the states of the mass-spring system via a two-link planar robot that transitions from noncontact to contact with the mass-spring through an impact collision. An additional objective is to limit the impact force to prevent damage to the robot or the environment (i.e., the mass-spring system). A regulation error, denoted by $e(t) \in \mathbb{R}^{3}$, is defined to quantify this objective as

$$
e=\left[\begin{array}{ll}
e_{m} & e_{r}^{\mathrm{T}}
\end{array}\right]^{\mathrm{T}}
$$

where $e_{r}(t) \triangleq\left[e_{r 1}(t), e_{r 2}(t)\right]^{\mathrm{T}} \in \mathbb{R}^{2}$ and $e_{m}(t) \in \mathbb{R}$ denote the regulation error for the end point of the second link of the robot and mass-spring system (see Fig. 1), respectively, and are defined as

$$
e_{r}=x_{r d}-x_{r} \quad e_{m}=x_{m d}-x_{m} .
$$

In (16), $x_{m d} \in \mathbb{R}$ denotes the constant known desired position of the mass, and $x_{r d}(t) \triangleq\left[x_{r d 1}(t), x_{r d 2}\right]^{\mathrm{T}} \in \mathbb{R}^{2}$ denotes the desired position of the end point of the second link of the robot. The subsequent development is based on the assumption that $q(t), \dot{q}(t), x_{m}(t)$, and $\dot{x}_{m}(t)$ are measurable and that $x_{r}(t)$ and $\dot{x}_{r}(t)$ can be obtained from $q(t)$ and $\dot{q}(t)$, respectively. To facilitate the subsequent control design and stability analysis, filtered tracking errors, denoted by $\eta_{m}(t) \in \mathbb{R}$ and $r_{r}(t) \in \mathbb{R}^{2}$, are defined as [47]

$$
\begin{aligned}
\eta_{m} & =\dot{e}_{m}+\alpha_{1} \tanh \left(e_{m}\right)+\alpha_{2} \tanh \left(e_{f}\right) \\
r_{r} & =\dot{e}_{r}+\alpha e_{r}
\end{aligned}
$$

where $\alpha, \alpha_{1}$, and $\alpha_{2} \in \mathbb{R}$ are positive constant gains and $e_{f}(t) \in$ $\mathbb{R}$ is an auxiliary filter variable designed as [47]

$$
\dot{e}_{f}=-\alpha_{3} \tanh \left(e_{f}\right)+\alpha_{2} \tanh \left(e_{m}\right)-k_{1} \cosh ^{2}\left(e_{f}\right) \eta_{m}
$$

where $k_{1} \in \mathbb{R}$ is a positive constant control gain and $\alpha_{3} \in \mathbb{R}$ is a positive constant filter gain. The filtered tracking error $r_{r}(t)$ is introduced to reduce the terms in the Lyapunov analysis (i.e., $r_{r}(t)$ can be used in lieu of including both $e_{r}(t)$ and $\dot{e}_{r}(t)$ in the stability analysis). The filtered tracking error $\eta_{m}(t)$ and the auxiliary signal $e_{f}(t)$ are introduced to eliminate a dependence on acceleration in the subsequently designed force controller [46].

\section{B. Closed-loop Error System}

By taking the time derivative of $m \eta_{m}(t)$ and utilizing (6), (7), (16), and (17), the following open-loop error system can be obtained:

$$
\begin{aligned}
m \dot{\eta}_{m}= & Y_{d} \theta_{d}-K_{I} \Lambda\left(x_{r 1}-x_{m}\right) \\
& +\alpha_{2} m \cosh ^{-2}\left(e_{f}\right) \dot{e}_{f}+\alpha_{1} m \cosh ^{-2}\left(e_{m}\right) \dot{e}_{m} .
\end{aligned}
$$


In (19), $Y_{d}\left(x_{m}\right)=\left(x_{m}-x_{o}\right)$ and $\theta_{d}=k_{s}$. To facilitate the subsequent analysis, the following notation is introduced [47]:

$$
Y_{d} \theta_{d}=Y_{d} K_{I} K_{I}^{-1} \theta_{d}=Y_{d k} \theta_{d k}=K_{I}\left(x_{m}-x_{o}\right)\left[\frac{k_{s}}{K_{I}}\right] .
$$

After using (17) and (18), the expression in (19) can be rewritten as

$$
\begin{aligned}
m \dot{\eta}_{m}=Y_{d} \theta_{d}+ & K_{I}\left(x_{r d 1}-\Lambda x_{r 1}\right) \\
& +K_{I} \Lambda x_{m}-K_{I} x_{r d 1}+\chi-\alpha_{2} m k_{1} \eta_{m}
\end{aligned}
$$

where $\chi\left(e_{m}, e_{f}, \eta_{m}, t\right) \in \mathbb{R}$ is an auxiliary term defined as

$$
\begin{aligned}
\chi= & \alpha_{1} m \cosh ^{-2}\left(e_{m}\right)\left(\eta_{m}-\alpha_{1} \tanh \left(e_{m}\right)\right) \\
& -\alpha_{1} \alpha_{2} m \cosh ^{-2}\left(e_{m}\right) \tanh \left(e_{f}\right) \\
& +\alpha_{2} m \cosh ^{-2}\left(e_{f}\right)\left(-\alpha_{3} \tanh \left(e_{f}\right)\right) \\
& +\alpha_{2} m \cosh ^{-2}\left(e_{f}\right)\left(\alpha_{2} \tanh \left(e_{m}\right)\right) .
\end{aligned}
$$

The motivation for the introduction of the filter signals $\eta_{m}(t)$ and $e_{f}(t)$ and the selective grouping of the terms in (22) allow the development of the following linear inequality (versus a quadratic inequality):

$$
|\chi| \leq \zeta_{1}\|z\|
$$

where $\zeta_{1} \in \mathbb{R}$ is a positive bounding constant and $z(t) \in \mathbb{R}^{3}$ is defined as

$$
z=\left[\begin{array}{lll}
\eta_{m} & \tanh \left(e_{m}\right) & \tanh \left(e_{f}\right)
\end{array}\right] .
$$

That is, the use of hyperbolic functions in the development of $\eta_{m}(t)$ and $e_{f}(t)$ allow the linear inequality in (23) to be developed; without the hyperbolic functions, the bound would be quadratic. Feedback terms in the controller can be used to damp out terms that are bounded by a linear function of the states without restricting the domain of the stability result as demonstrated in the subsequent stability analysis. If the hyperbolic terms had not been used in the filter structure, the bound in (23) would have been quadratic, potentially limiting the domain of the stability result (e.g., a semiglobal stability result).

Based on (21) and the subsequent stability analysis, the desired robot link position is designed as

$x_{r d 1}=Y_{d} \hat{\theta}_{d k}+x_{m}+k_{2} \tanh \left(e_{m}\right)-k_{1} k_{2} \cosh ^{2}\left(e_{f}\right) \tanh \left(e_{f}\right)$

$x_{r d 2}=\varepsilon$.

In (25), $\varepsilon \in \mathbb{R}$ is an appropriate positive constant (i.e., $\varepsilon$ is selected; therefore, the robot will impact the mass-spring system in the vertical direction), $k_{2} \in \mathbb{R}$ is a positive constant control gain, and the control gain $k_{1} \in \mathbb{R}$ is defined as

$$
k_{1}=\frac{1}{m_{l}}\left(3+k_{n 1} \zeta_{1}^{2}\right)
$$

where $k_{n 1} \in \mathbb{R}$ is a positive constant nonlinear damping gain. The parameter estimate $\hat{\theta}_{d k}(t) \in \mathbb{R}$ in (25) is generated by the adaptive update law

$$
\dot{\hat{\theta}}_{d k}=\operatorname{proj}\left(\Gamma Y_{d} \eta_{m}\right) .
$$

In (27), $\Gamma \in \mathbb{R}$ is a positive constant, and $\operatorname{proj}(\cdot)$ denotes a sufficiently smooth projection algorithm [48] utilized to guarantee that $\hat{\theta}_{d k}(t)$ can be bounded as

$$
\underline{\theta}_{d k} \leq \hat{\theta}_{d k} \leq \bar{\theta}_{d k}
$$

where $\underline{\theta}_{d k}$ and $\bar{\theta}_{d k} \in \mathbb{R}$ denote known constant lower and upper bounds for $\theta_{d k}(t)$, respectively.

After substituting (25) into (21), the closed-loop error system for $\eta_{m}(t)$ can be obtained as

$$
\begin{aligned}
m \dot{\eta}_{m}= & K_{I}\left(x_{r d 1}-\Lambda x_{r 1}\right)+K_{I}\left(\Lambda x_{m}-x_{m}\right) \\
& +K_{I} k_{1} k_{2} \cosh ^{2}\left(e_{f}\right) \tanh \left(e_{f}\right) \\
& +Y_{d k} \tilde{\theta}_{d k}-K_{I} k_{2} \tanh \left(e_{m}\right)+\chi-\alpha_{2} m k_{1} \eta_{m} .
\end{aligned}
$$

In (29), the parameter estimation error $\tilde{\theta}_{d k}(t) \in \mathbb{R}$ is defined as

$$
\tilde{\theta}_{d k}=\theta_{d k}-\hat{\theta}_{d k} .
$$

The open-loop robot error system can be obtained by taking the time derivative of $r_{r}(t)$ and premultiplying by the robot inertia matrix as

$$
\bar{M} \dot{r}_{r}=Y_{r} \theta_{r}-\bar{C} r_{r}-F
$$

where (5), (16), and (17) were utilized, and

$$
\begin{aligned}
Y_{r} \theta_{r}=\bar{M} \ddot{x}_{r d}+\alpha \bar{M} \dot{e}_{r}+\bar{h}+\bar{C} \dot{x}_{r d}+\alpha \bar{C} x_{r d} \\
+\left[\begin{array}{c}
K_{I} \Lambda\left(x_{r 1}-x_{m}\right) \\
0
\end{array}\right]-\alpha \bar{C} x_{r}
\end{aligned}
$$

where $Y_{r}\left(x_{r}, \dot{x}_{r}, x_{m}, \dot{x}_{m}, e_{f}, \eta_{m}, t\right) \in \mathbb{R}^{2 \times P}$ denotes a known regression matrix and $\theta_{r} \in \mathbb{R}^{P}$ denotes an unknown constant parameter vector. By making substitutions from the dynamic model and the previous error systems, $\ddot{x}_{r d}(t)$ can be expressed without a dependence on acceleration terms (see Appendix A). Based on (30) and the subsequent stability analysis, the robot force control input is designed as

$$
F=Y_{r} \hat{\theta}_{r}+\operatorname{Tanh}\left(e_{r}\right)+k_{3} \operatorname{Tanh}\left(r_{r}\right)
$$

where $k_{3} \in \mathbb{R}$ is a positive constant control gain and $\hat{\theta}_{r}(t) \in \mathbb{R}^{P}$ is an estimate for $\theta_{r}$ generated by the following adaptive update law:

$$
\dot{\hat{\theta}}_{r}=\operatorname{proj}\left(\Gamma_{r} Y_{r}^{\mathrm{T}} r_{r}\right) .
$$

In (33), $\Gamma_{r} \in \mathbb{R}^{P \times P}$ is a positive definite constant diagonal adaptation gain matrix, and $\operatorname{proj}(\cdot)$ denotes a projection algorithm utilized to guarantee that the $i$ th element of $\hat{\theta}_{r}(t)$ can be bounded as

$$
\underline{\theta}_{r i} \leq \hat{\theta}_{r i} \leq \bar{\theta}_{r i}
$$

where $\underline{\theta}_{r i}$ and $\bar{\theta}_{r i} \in \mathbb{R}$ denote known constant lower and upper bounds for each element of $\theta_{r}(t)$, respectively.

The closed-loop error system for $r_{r}(t)$ can be obtained after substituting (32) into (30) as

$$
\bar{M} \dot{r}_{r}=Y_{r} \tilde{\theta}_{r}-\bar{C} r_{r}-\operatorname{Tanh}\left(e_{r}\right)-k_{3} \operatorname{Tanh}\left(r_{r}\right) .
$$

In (35), the parameter estimation error $\tilde{\theta}_{r}(t) \in \mathbb{R}^{P}$ is defined as

$$
\tilde{\theta}_{r}=\theta_{r}-\hat{\theta}_{r} .
$$




\section{STABiLity ANALYSiS}

Theorem 1: The controller given by (25), (27), (32), and (33) ensures the semiglobal asymptotic regulation of the MSR system in the sense that

$$
\left|e_{m}(t)\right| \rightarrow 0 \quad\left\|e_{r}(t)\right\| \rightarrow 0 \quad \text { as } t \rightarrow \infty
$$

provided that $k_{1}$ and $k_{2}$ are selected properly (see Appendix B) and the following sufficient gain conditions are satisfied:

$$
\begin{aligned}
k_{n 1} & >\frac{1}{4 \alpha_{2} \min \left\{\alpha_{1} k_{2} \underline{\zeta}_{K}, \alpha_{3} k_{2} \underline{\zeta}_{K}, \alpha_{2}\right\}} \\
\alpha_{2} & >\max \left\{\frac{1}{4 \alpha}, \frac{\zeta_{x_{m}}^{2}}{\lambda}\right\} \bar{\zeta}_{K}^{2} \\
k_{3} \alpha & >\left(\sqrt{\frac{2 V_{u}}{a_{1}}}+1\right)^{2}
\end{aligned}
$$

where $V_{u}$ and $\lambda \in \mathbb{R}$ are known positive constants (see Appendix B) and $a_{1}, \alpha, \alpha_{1}, \alpha_{2}, \alpha_{3}, k_{n 1}, k_{2}, k_{3}, \zeta_{x_{m}}, \zeta_{x_{r}}, \underline{\zeta}_{K}$, and $\bar{\zeta}_{K}$ are defined in (4), (9), (11), (17), (18), (25), (26), and (32), respectively.

Proof: See Appendix B.

Remark 3: The sufficient gain condition in (38) indicates that, as $K_{I}$ becomes infinitely large, $\alpha_{2}$ must also grow infinitely large. See the classic discussion on this issue given in [33]. In this result, we only consider contact with surfaces with finite $K_{I}$. In the experimental results for this paper, the actual values for $\alpha_{2}$ were selected much lower than the sufficient condition indicated in (38), as is typical in Lyapunov-based designs.

\section{EXPERIMENTAL RESULTS}

The testbed shown in Figs. 2 and 3 was developed for the experimental demonstration of the proposed controller. The testbed is composed of a mass-spring system and a two-link planar robot. The body of the mass-spring system includes a U-shaped aluminum plate (item (8) in Fig. 2) mounted on an undercarriage with porous carbon air bearings which enables the undercarriage to glide on an air cushion over a glass-covered aluminum rail. A steel-core spring (item (1) in Fig. 2) connects the $\mathrm{U}$-shaped aluminum plate to an aluminum frame, and a linear variable displacement transducer (LVDT) (item (2) in Fig. 2) is used to measure the position of the undercarriage assembly. The impact surface consists of an aluminum plate connected to the undercarriage assembly through a stiff spring mechanism (item (7) in Fig. 2). A capacitance probe (item (3) in Fig. 2) is used to measure the deflection of the stiff spring mechanism. The two-link planar robot (items (4-6) in Fig. 2) is made of two aluminum links, mounted on 240.0(base link) and 20.0- Nm (second link) direct-drive switched reluctance motors. The motors are controlled through power electronics operating in torque control mode. The motor resolvers provide rotor-position measurements with a resolution of 614400 pulses/revolution, and a standard backward-difference algorithm is used to determine numerically the angular velocity from the encoder readings. A Pentium 2.8-GHz PC operating under QNX hosts the control algorithm, which was implemented via a custom graphical user interface to facilitate

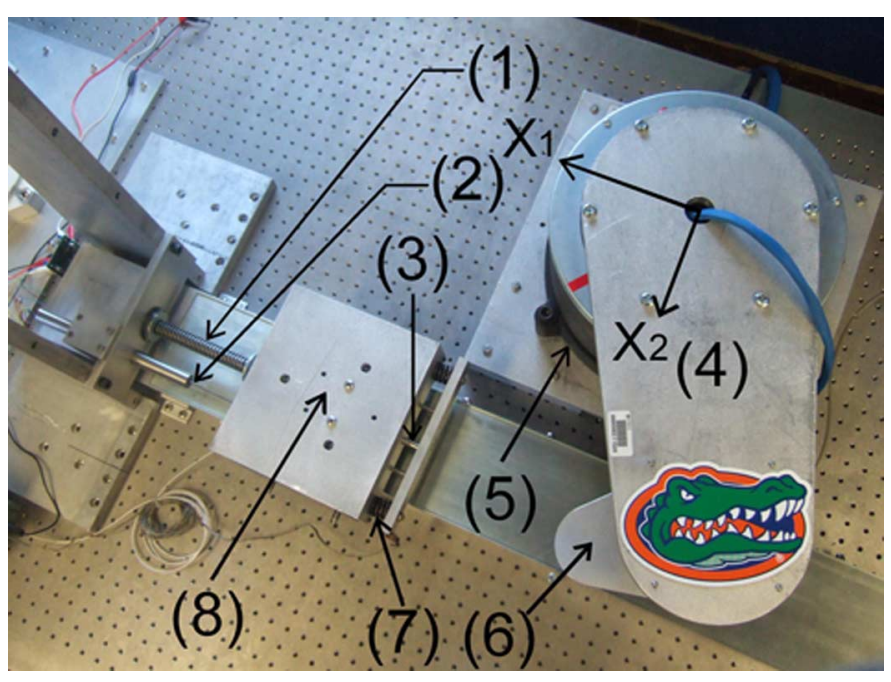

Fig. 2. Top view of the experimental testbed including the following: (1) Spring, (2) LVDT, (3) capacitance probe, (4) link1, (5) motor1, (6) link2, (7) stiff spring mechanism, and (8) mass.

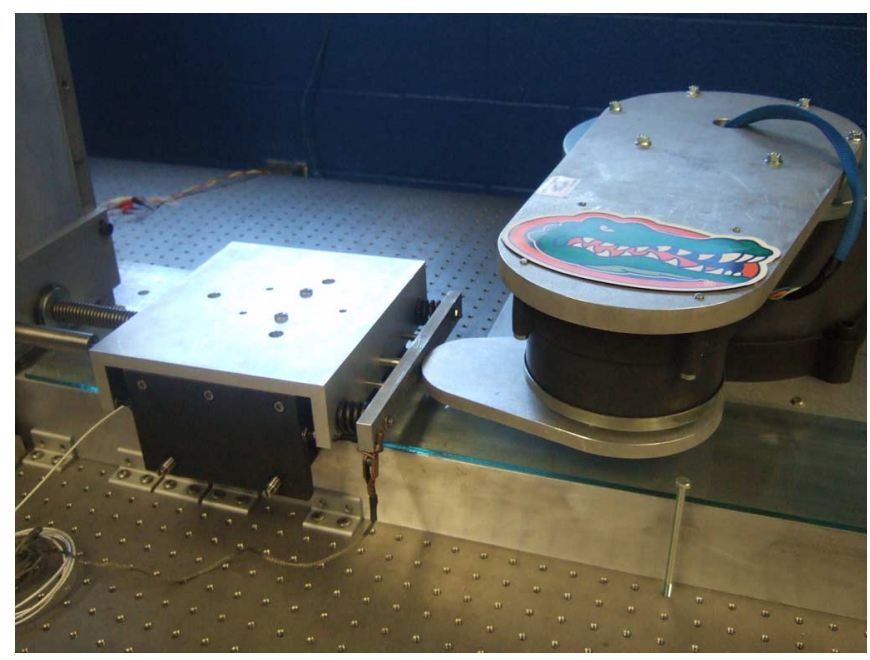

Fig. 3. Side view of the experimental testbed.

real-time graphing, data logging, and the ability to adjust control gains without recompiling the program. Data acquisition and control implementation were performed at a frequency of $2.0 \mathrm{kHz}$ using the ServoToGo I/O board.

\section{A. Experiment 1}

The first experiment was conducted with the controller proposed in this paper where the control input takes the form in (32). The initial conditions for the robot coordinates and the mass-spring position were (in meters)

$$
\left[x_{r 1}(0) \quad x_{r 2}(0) \quad x_{m}(0)\right]=\left[\begin{array}{lll}
0.060 & 0.436 & 0.206
\end{array}\right] .
$$

The initial velocity of the robot and mass-spring were zero, and the desired mass-spring position was (in meters)

$$
x_{m d}=0.236 .
$$

That is, the tip of the second link of the robot was initially 176 $\mathrm{mm}$ from the desired setpoint and $146 \mathrm{~mm}$ from $x_{0}$ along the 
(a)
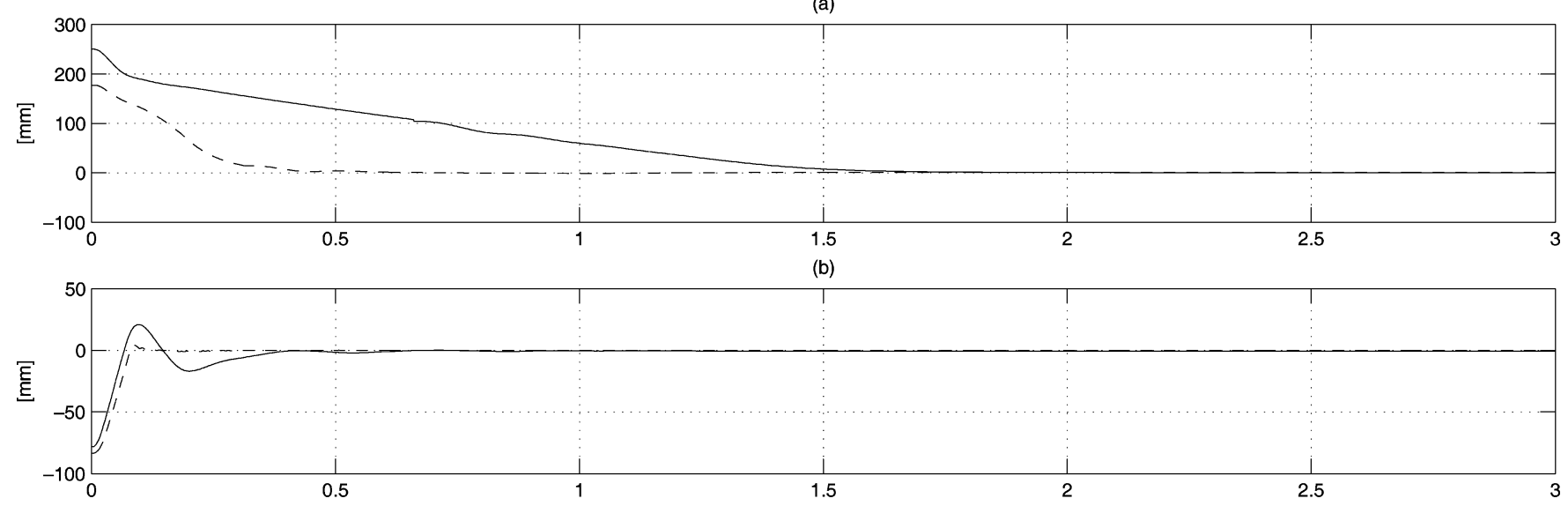

(c)

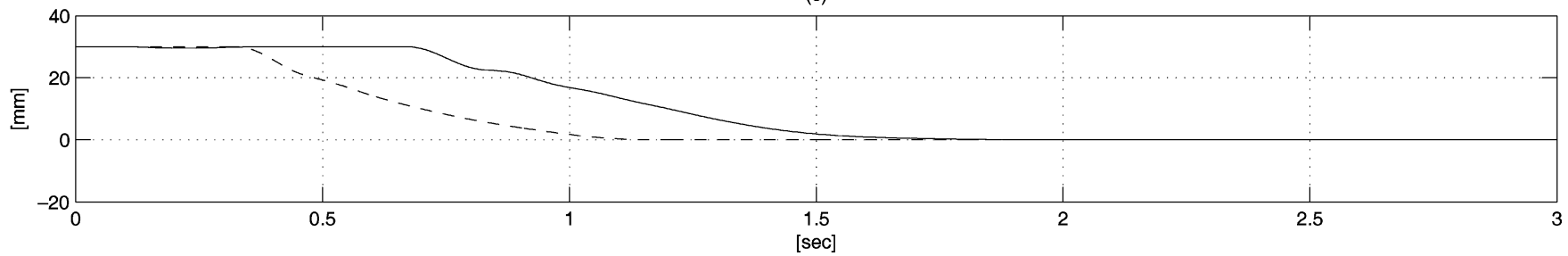

Fig. 4. Mass-spring and robot errors $e(t)$ with (solid line) the controller in (32) and with (dashed line) the controller in [28]. Plot (a) indicates the position error of the robot tip along the $X_{1}$-axis [i.e., $\left.e_{r 1}(t)\right],(\mathrm{b})$ indicates the position error of the robot tip along the $X_{2}$-axis [i.e., $e_{r 2}(t)$ ], and (c) indicates the position error of the mass-spring [i.e., $\left.e_{m}(t)\right]$.

$X_{1}$-axis (see Fig. 2). Once the initial impact occurs, the robot is required to depress the spring (item (1) in Fig. 2) to move the mass of $30 \mathrm{~mm}$ along the $X_{1}$-axis.

The control gains $\alpha$ and $k_{3}$, defined as scalars in (17) and (32), were implemented (with nonconsequential implications to the stability result) as diagonal gain matrices to provide more flexibility in the experiment. Specifically, the control gains were selected as

$$
\begin{array}{lll}
k_{1}=2 & k_{2}=0.47 & k_{3}=\operatorname{diag}\{95,5\} \\
\alpha_{1}=55 & \alpha_{2}=15 & \alpha_{3}=0.1 \quad \alpha=\operatorname{diag}\{50,4.5\} .
\end{array}
$$

The adaptation gains were selected as

$$
\begin{gathered}
\begin{aligned}
& \Gamma=1 \\
& \Gamma_{r}=\operatorname{diag}\left\{2 \times 10^{9}, 2.5 \times 10^{4}, 0.2,4 \times 10^{8}, 1.5 \times 10^{4},\right. \\
&\left.\quad 0.1,6 \times 10^{7}, 1 \times 10^{3}, 0.01,1 \times 10^{9}\right\} .
\end{aligned}
\end{gathered}
$$

The adaptation gains $\Gamma_{r}$ in (43) are used to enable the adaptive estimate to be sufficiently changed relative to the large values of the uncertain parameters in $\theta_{r}$ due to the impact stiffness terms.

The mass-spring and robot errors [i.e., $e(t)]$ are shown in Fig. 4. The peak steady-state position error of the end point of the second link of the robot along the $X_{1}$-axis (i.e., $\left|e_{r 1}(t)\right|$ ) and along the $X_{2}$-axis (i.e., $\left.\left|e_{r 2}(t)\right|\right)$ are 0.216 and $0.737 \mathrm{~mm}$, respectively. The peak steady-state position error of the mass (i.e., $\left.\left|e_{m}(t)\right|\right)$ is $2.56 \mu \mathrm{m}$. The relatively large $\left|e_{r 1}(t)\right|$ is due to the mismatch between the estimate value $\hat{\theta}_{d k}(t)$ and the real value $\theta_{d k}(t)$ in $e_{r 1}(t)$. The relatively large $\left|e_{r 2}(t)\right|$ is due to the inability of the model to capture friction and slipping effects on the contact surface. In this experiment, a significant friction is present along the $X_{2}$-axis between the robot tip and the contact
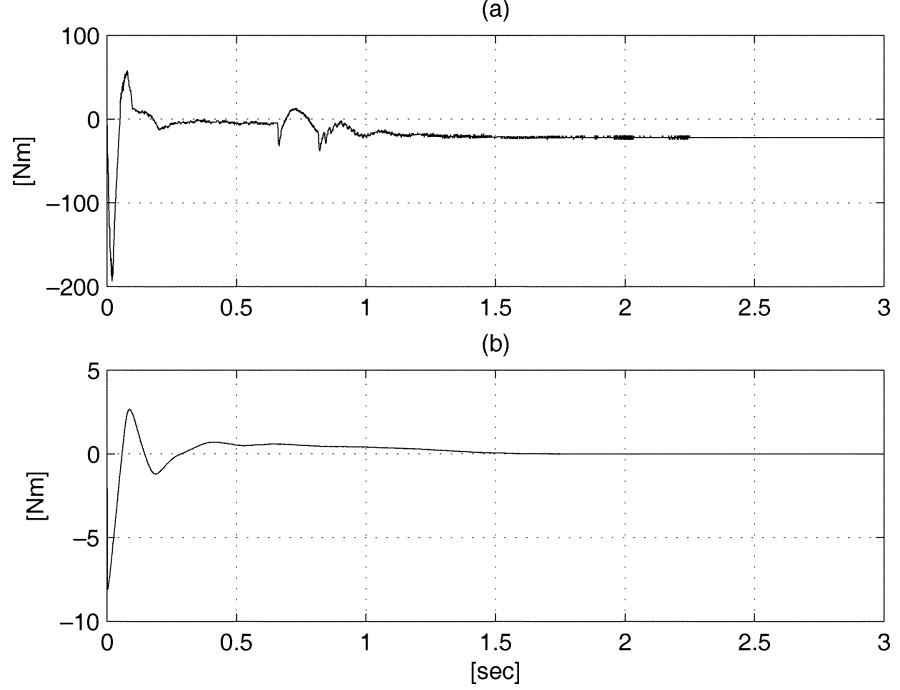

Fig. 5. Applied control torques $J^{\mathrm{T}}(q) F(t)$, with controller in (32), for the (a) base motor and (b) second link motor.

surface due to a large normal spring force applied along the $X_{1}$-axis.

The input control torques [i.e., $J^{\mathrm{T}}(q) F(t)$ ] are shown in Fig. 5. The resulting desired trajectory along the $X_{1}$-axis [i.e., $\left.x_{r d 1}(t)\right]$ is shown in Fig. 6, and the desired trajectory along the $X_{2}$-axis was chosen as $x_{r d 2}=0.358 \mathrm{~m}$.

\section{B. Experiment 2}

The second experiment was conducted with the same adaptive controller without saturating the feedback terms [28]

$$
F=Y_{r} \hat{\theta}_{r}+e_{r}+k_{3} r_{r} .
$$




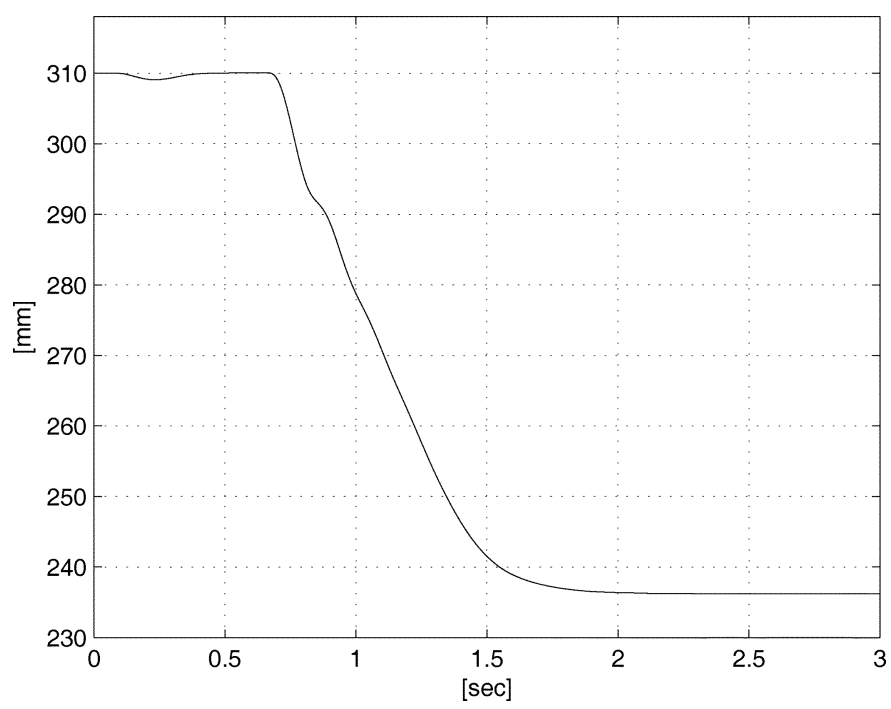

Fig. 6. Computed desired robot trajectory $x_{r d 1}(t)$.

The initial conditions for the robot and the mass-spring were chosen to be the same as that in (40). The initial velocity of the robot and mass-spring were zero, and the desired mass-spring position was the same as in (41). It was found that, with the same control gains as in (42) and (43), the impact force was so large that the aluminum plate was flexed to the point of contact with the capacitance probe. On artificially saturating the control inputs to the level that the impact force is acceptable, it was found that the control inputs were not enough to drive the mass-spring to the desired position. Hence, the control gains needed to be tuned again to ensure a satisfactory performance. They were chosen as

$$
\begin{aligned}
& k_{1}=0.22 \quad k_{2}=2.945 \quad k_{3}=\operatorname{diag}\{185,175\} \\
& \alpha_{1}=75 \quad \alpha_{2}=100 \quad \alpha_{3}=0.001 \quad \alpha=\operatorname{diag}\{80,100\} .
\end{aligned}
$$

The adaptation gains were selected as

$$
\begin{aligned}
& \Gamma=0.1 \\
& \Gamma_{r}=\operatorname{diag}\left\{7 \times 10^{10}, 2 \times 10^{6}, 0.2,1 \times 10^{10}, 5 \times 10^{5},\right. \\
&\left.\quad 0.1,1 \times 10^{10}, 1 \times 10^{5}, 0.06,4 \times 10^{10}\right\} .
\end{aligned}
$$

The mass-spring and robot errors [i.e., $e(t)]$ are shown in Fig. 4. The peak steady-state position error of the end point of the second link of the robot along the $X_{1}$-axis (i.e., $\left|e_{r 1}(t)\right|$ ) and along the $X_{2}$-axis (i.e., $\left.\left|e_{r 2}(t)\right|\right)$ are 0.376 and $0.075 \mathrm{~mm}$, respectively. The peak steady-state position error of the mass (i.e., $\left.\left|e_{m}(t)\right|\right)$ is $5 \mu \mathrm{m}$.

The computed and actual control inputs which were artificially saturated are provided in Figs. 7 and 8, respectively.

\section{Discussion}

Figs. 4, 5, and 7 show that, for the same initial conditions and comparable steady-state error values, the control input with the controller in [28] has to be artificially saturated to limit the forces during impact, whereas no such artificial saturation was required with the controller in (32). The fact that the saturation
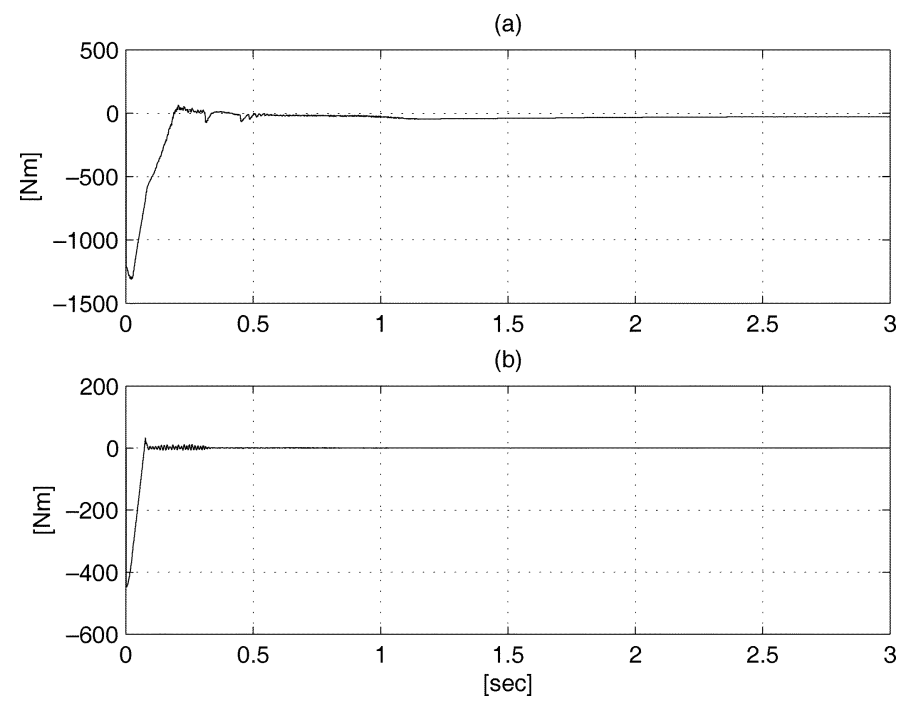

Fig. 7. Computed control torques $J^{\mathrm{T}}(q) F(t)$ for the (a) base motor and (b) second link motor for the controller in [28].

(a)

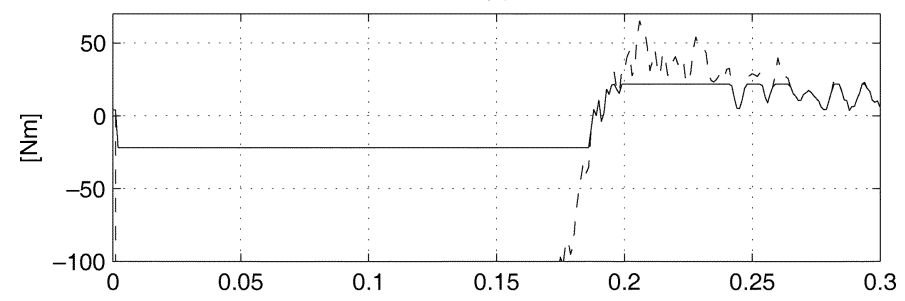

(b)

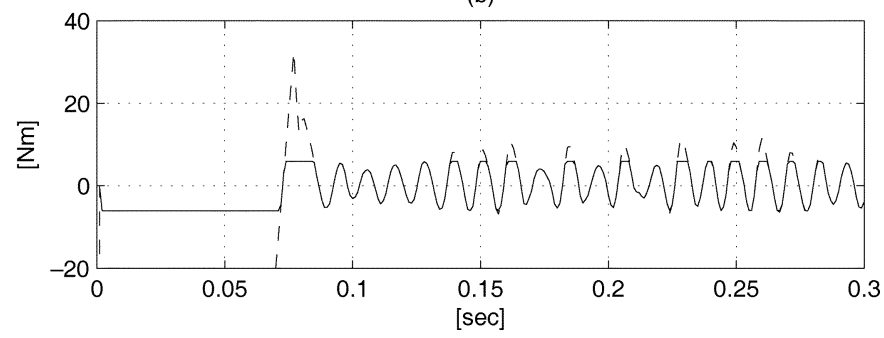

Fig. 8. (Solid line) Applied control torques $J^{\mathrm{T}}(q) F(t)$ versus (dashed line) computed control torques for the (a) base motor and (b) second link motor for the controller in [28].

of the feedback terms of the controller in (32) is included in the stability analysis is significant because the artificial saturation of the computed torques in Figs. 7 and 8 may lead to instabilities. The tradeoff for saturating the torques is that the controller in [28] exhibits a reduced transient performance despite the fact that the artificial saturation threshold is below the saturation level of the controller in (32). The improved transient performance of the controller in [28] is due to the feedback gains multiplied directly by the state, versus the feedback gains multiplied by saturated trigonometric functions of the state as in (32). For applications where the initial conditions are minimal or large impact forces are acceptable, the controller in [28] may provide the benefit of an improved transient performance; however, for applications that may have large errors or where impact forces must be mitigated, the controller in (32) provides the advantage of limited actuation with proven stability. 


\section{CONCLUSION}

In this paper, we consider a two-link planar robotic arm that transitions from free motion to contact with an unactuated mass-spring system. An adaptive nonlinear Lyapunov-based controller with bounded torque input amplitudes is proven to regulate the states of the system. The feedback elements for the controller in this paper are contained inside hyperbolic tangent functions as a means to limit the impact forces resulting from large initial conditions as the robot transitions from noncontact to contact. The continuous controller yields a semiglobal asymptotic regulation of the mass-spring and robot links. In comparison with the previous result [28], where the feedback terms of the controller were not saturated, the controller in this paper obtained a considerable reduction in the control effort while achieving comparable steady-state error values. Experimental results are provided to illustrate the successful performance of the controller.

\section{APPENDIX A}

\section{EXPRESSION OF $\ddot{x}_{r d 1}$}

Since $x_{r d 2}$ is a constant, the subsequent development is only focused on determining $\ddot{x}_{r d 1}(t)$. After using (16), (18), (25), and (27), the first time derivative of $x_{r d 1}(t)$ can be determined as

$$
\begin{aligned}
\dot{x}_{r d 1}= & Y_{d}\left(\operatorname{proj}\left(\Gamma Y_{d} \eta_{m}\right)\right)+\left(\hat{\theta}_{d k}+1-k_{2} \cosh ^{-2}\left(e_{m}\right)\right) \dot{x}_{m} \\
& -k_{1} k_{2}\left(\sinh ^{2}\left(e_{f}\right)+\cosh ^{2}\left(e_{f}\right)\right) \\
& \times\left(-\alpha_{3} \tanh \left(e_{f}\right)+\alpha_{2} \tanh \left(e_{m}\right)-k_{1} \cosh ^{2}\left(e_{f}\right) \eta_{m}\right) .
\end{aligned}
$$

Based on the fact that the projection algorithm for $\dot{\hat{\theta}}_{d k}(t)$ is designed to be sufficiently smooth [48], the expressions in (27) and (45) can be used to determine the second time derivative of $x_{r d 1}(t)$ as

$$
\begin{aligned}
\ddot{x}_{r d 1}= & Y_{d} \frac{\partial\left(\operatorname{proj}\left(\Gamma Y_{d} \eta_{m}\right)\right)}{\partial \eta_{m}} \dot{\eta}_{m} \\
& +\left(Y_{d} \frac{\partial\left(\operatorname{proj}\left(\Gamma Y_{d} \eta_{m}\right)\right)}{\partial x_{m}}+2 \operatorname{proj}\left(\Gamma Y_{d} \eta_{m}\right)\right) \dot{x}_{m} \\
& -2 k_{2} \cosh ^{-3}\left(e_{m}\right) \sinh \left(e_{m}\right) \dot{x}_{m}^{2} \\
& +\left(\hat{\theta}_{d k}+1-k_{2} \cosh ^{-2}\left(e_{m}\right)\right) \ddot{x}_{m} \\
& -4 k_{1} k_{2}\left(\sinh ^{2}\left(e_{f}\right) \cosh \left(e_{f}\right)\right) \dot{e}_{f}^{2} \\
& -k_{1} k_{2}\left(\sinh ^{2}\left(e_{f}\right)+\cosh ^{2}\left(e_{f}\right)\right) \\
& \cdot\left(-\alpha_{3} \cosh ^{-2}\left(e_{f}\right)-2 k_{1} \cosh \left(e_{f}\right) \sinh \left(e_{f}\right) \eta_{m}\right) \dot{e}_{f} \\
& +k_{1} k_{2}\left(\sinh ^{2}\left(e_{f}\right)+\cosh ^{2}\left(e_{f}\right)\right) \\
& \cdot\left(-\alpha_{2} \cosh ^{-2}\left(e_{m}\right) \dot{e}_{m}+k_{1} \cosh ^{2}\left(e_{f}\right) \dot{\eta}_{m}\right)
\end{aligned}
$$

After substituting (18) and (19) into (46) for $\dot{e}_{f}(t)$ and $\dot{\eta}_{m}(t)$, respectively, and substituting (6) and (7) into (46) for $\ddot{x}_{m}(t)$, the expression for $\bar{M}\left(x_{r}\right) \ddot{x}_{r d}(t)$ in the linear parameterization in (31) can be determined without requiring acceleration measurements.

\section{APPENDIX B \\ STABILITY ANALYSIS PROOF}

In the following proof, a Lyapunov function and its derivative are provided. The analysis is then separated into two cases: contact and noncontact. For the noncontact case, the stability analysis indicates that the controller and error signals are bounded and converge to an arbitrarily small region where contact must occur. When contact occurs, a Lyapunov analysis is provided, which illustrates that the MSR system asymptotically converges to the desired setpoint.

Proof: Let $V(t) \in \mathbb{R}$ denote the following nonnegative radially unbounded function (i.e., a Lyapunov function candidate):

$$
\begin{aligned}
V= & \frac{1}{2} r_{r}^{\mathrm{T}} \bar{M} r_{r}+\frac{1}{2} \tilde{\theta}_{r}^{\mathrm{T}} \Gamma_{r}^{-1} \tilde{\theta}_{r}+\frac{1}{2} \tilde{\theta}_{d k}^{\mathrm{T}} K_{I} \Gamma^{-1} \tilde{\theta}_{d k} \\
& +k_{2} K_{I}\left[\ln \left(\cosh \left(e_{m}\right)\right)+\ln \left(\cosh \left(e_{f}\right)\right)\right] \\
& +e_{r}^{\mathrm{T}} e_{r}+\frac{1}{2} m \eta_{m}^{2}+\ln \left(\cosh \left(e_{r 1}\right)\right)+\ln \left(\cosh \left(e_{r 2}\right)\right)
\end{aligned}
$$

where (9) and (14) can be utilized to bound $V(t)$ as

$$
\frac{a_{1}}{2}\left\|r_{r}\right\|^{2} \leq V \leq \lambda_{1}\|\bar{z}\|^{2}+\zeta_{\theta}
$$

where $a_{1} \in \mathbb{R}$ is defined in (9) and $\lambda_{1}, \zeta_{\theta} \in \mathbb{R}$, and $\bar{z} \in \mathbb{R}^{7}$ are defined as

$$
\begin{aligned}
\lambda_{1} & =\max \left\{\frac{a_{2}}{2}, 1, \frac{m_{u}}{2}, k_{2} \bar{\zeta}_{K}\right\} \\
\zeta_{\theta} & =\frac{1}{2} \bar{\zeta}_{K} \Gamma^{-1} \zeta_{\theta_{d k}}^{2}+\frac{1}{2} \lambda_{\max }\left\{\Gamma_{r}^{-1}\right\}\left\|\zeta_{\theta_{r}}\right\|^{2} \\
\bar{z} & =\left[r_{r}^{\mathrm{T}}, e_{r}^{\mathrm{T}}, \eta_{m}, e_{m}, e_{f}\right]^{\mathrm{T}}
\end{aligned}
$$

where $\lambda_{\max }\{\cdot\} \in \mathbb{R}$ denotes the maximum eigenvalue of a matrix and $\zeta_{\theta_{d k}}$ and $\left\|\zeta_{\theta_{r}}\right\|$ are the known upper bounds of $\tilde{\theta}_{d k}(t)$ and $\left\|\tilde{\theta}_{r}(t)\right\|$, respectively. After using (10), (15), (17), (18), (26), (27), (29), (33), and (35), the time derivative of (47) can be determined as

$$
\begin{aligned}
\dot{V} \leq & -k_{3} \tanh ^{2}\left(\left\|r_{r}\right\|\right)-\alpha_{1} k_{2} K_{I} \tanh ^{2}\left(e_{m}\right) \\
& -\alpha_{3} k_{2} K_{I} \tanh ^{2}\left(e_{f}\right)+2 e_{r}^{\mathrm{T}} r_{r}-2 \alpha e_{r}^{\mathrm{T}} e_{r} \\
& -3 \alpha_{2} \eta_{m}^{2}-k_{n 1} \zeta_{1}^{2} \alpha_{2} \eta_{m}^{2}-\alpha \tanh ^{2}\left(\left\|e_{r}\right\|\right) \\
& +\eta_{m}\left[K_{I}\left(x_{r d 1}-\Lambda x_{r 1}\right)+K_{I}\left(\Lambda x_{m}-x_{m}\right)+\chi\right](50)
\end{aligned}
$$

The expression in (50) will now be examined under two different scenarios.

Case 1-Noncontact: For this case, the systems are not in contact $(\Lambda=0)$, and (50) can be rewritten as

$$
\begin{aligned}
\dot{V} \leq & -k_{3} \tanh ^{2}\left(\left\|r_{r}\right\|\right)-\alpha_{1} k_{2} K_{I} \tanh ^{2}\left(e_{m}\right)-\alpha_{3} k_{2} K_{I} \tanh ^{2}\left(e_{f}\right) \\
& +2 e_{r}^{\mathrm{T}} r_{r}-2 \alpha e_{r}^{\mathrm{T}} e_{r}-3 \alpha_{2} \eta_{m}^{2}-k_{n 1} \zeta_{1}^{2} \alpha_{2} \eta_{m}^{2} \\
& -\alpha \tanh ^{2}\left(\left\|e_{r}\right\|\right)+\eta_{m}\left[K_{I} x_{r d 1}-K_{I} x_{m}+\chi\right]
\end{aligned}
$$


Based on (11), (16), and (23), the expression in (51) can be rewritten as

$$
\begin{aligned}
\dot{V} \leq & -k_{3} \tanh ^{2}\left(\left\|r_{r}\right\|\right)-\alpha_{1} k_{2} \underline{\zeta}_{K} \tanh ^{2}\left(e_{m}\right) \\
& -\alpha_{3} k_{2} \zeta_{K} \tanh ^{2}\left(e_{f}\right)-2 \alpha_{2} \eta_{m}^{2}-\alpha \tanh ^{2}\left(\left\|e_{r}\right\|\right) \\
& -\left[\alpha\left\|e_{r}\right\|^{2}-\bar{\zeta}_{K}\left|\eta_{m}\right|\left\|e_{r}\right\|\right] \\
& -\left[k_{n 1} \zeta_{1}^{2} \alpha_{2} \eta_{m}^{2}-\zeta_{1}\|z\| \eta_{m} \mid\right] \\
& -\left[\alpha_{2} \eta_{m}^{2}-\bar{\zeta}_{K}\left|\eta_{m} \| x_{m}-x_{r 1}\right|\right] \\
& -\left[\alpha\left\|e_{r}\right\|^{2}-2\left\|e_{r}\right\|\left\|r_{r}\right\|\right] .
\end{aligned}
$$

After completing the square on the last four lines, (52) can be expressed as

$$
\begin{aligned}
\dot{V} \leq & -\beta\|z\|^{2}-\alpha \tanh ^{2}\left(\left\|e_{r}\right\|\right)-\left(\alpha_{2}-\frac{\bar{\zeta}_{K}^{2}}{4 \alpha}\right) \eta_{m}^{2} \\
& +\frac{\bar{\zeta}_{K}^{2}\left(x_{m}-x_{r 1}\right)^{2}}{4 \alpha_{2}} \\
& -\left(k_{3}-\frac{\left\|r_{r}\right\|^{2}}{\alpha \tanh ^{2}\left(\left\|r_{r}\right\|\right)}\right) \tanh ^{2}\left(\left\|r_{r}\right\|\right)
\end{aligned}
$$

where $\beta \in \mathbb{R}$ is defined as

$$
\beta=\min \left\{\alpha_{1} k_{2} \underline{\zeta}_{K}, \alpha_{3} k_{2} \underline{\zeta}_{K}, \alpha_{2}\right\}-\frac{1}{4 \alpha_{2} k_{n 1}}>0
$$

provided $k_{n 1}$ is selected according to (37). Provided that $\alpha_{2}$ is selected according to the sufficient condition in (38), then (4), (13), (48), and the fact that

$$
\zeta_{x_{r}} \leq x_{r 1}<x_{m} \leq \zeta_{x_{m}}
$$

for the noncontact case can be used to rewrite (53) as

$$
\begin{array}{r}
\dot{V} \leq-\beta\|z\|^{2}-\alpha \tanh ^{2}\left(\left\|e_{r}\right\|\right)+\varepsilon_{x} \\
-\left(k_{3}-\frac{1}{\alpha}\left(\sqrt{\frac{2 V}{a_{1}}}+1\right)^{2}\right) \tanh ^{2}\left(\left\|r_{r}\right\|\right) .
\end{array}
$$

In (55), $\varepsilon_{x} \in \mathbb{R}$ is a known positive arbitrarily small constant that is defined as

$$
\varepsilon_{x}=\frac{\bar{\zeta}_{K}^{2} \zeta_{x_{m}}^{2}}{\alpha_{2}}
$$

Provided that the following sufficient condition is satisfied:

$$
k_{3} \alpha>\left(\sqrt{\frac{2 V}{a_{1}}}+1\right)^{2}
$$

the expression in (55) can be expressed as

$$
\dot{V} \leq-\lambda\|y\|^{2}+\varepsilon_{x}
$$

where $\lambda$ is a known constant and $y \in \mathbb{R}^{5}$ is defined as

$$
y=\left[\begin{array}{ll}
z^{\mathrm{T}} & \tanh \left(\left\|e_{r}\right\|\right) \quad \tanh \left(\left\|r_{r}\right\|\right)
\end{array}\right]^{\mathrm{T}} .
$$

In (57), $\varepsilon_{x}$ can be made arbitrarily small by making $\alpha_{2}$ large. Based on (47) and (57), if $\lambda\|y(t)\|^{2}>\varepsilon_{x}$, then Barbalat's lemma can be used to conclude that $\dot{V}(t) \rightarrow 0$ since $V(t)$ is lower bounded, $\dot{V}(t)$ is negative semidefinite, and $\dot{V}(t)$ can be shown to be uniformly continuous (UC). As $\dot{V}(t) \rightarrow 0$, eventually, $\lambda\|y(t)\|^{2} \leq \varepsilon_{x}$. While $\lambda\|y(t)\|^{2}>\varepsilon_{x}$, (47), (48), and (57) can be used to conclude that $V(t) \in \mathcal{L}_{\infty}$, and the sufficient condition given in (56) can be expressed as

$$
k_{3} \alpha>\left(\sqrt{\frac{2\left(\lambda_{1}\|\bar{z}(0)\|^{2}+\zeta_{\theta}\right)}{a_{1}}}+1\right)^{2} .
$$

Provided that $\alpha_{2}>\bar{\zeta}_{K}^{2} \zeta_{x_{m}}^{2} / \lambda$, then, eventually, $\lambda\|y(t)\|^{2} \leq$ $\varepsilon_{x}<\lambda$. Based on (24) and (58), the fact that $\lambda\|y(t)\|^{2} \leq \varepsilon_{x}<$ $\lambda$ can be used to conclude that $e_{m}(t), e_{f}(t), e_{r}(t), r_{r}(t)$, and $\eta_{m}(t) \in \mathcal{L}_{\infty}$. Since $\tilde{\theta}_{r}(t)$ and $\tilde{\theta}_{d k}(t) \in \mathcal{L}_{\infty}$ from the use of a projection algorithm, the previous facts can be used to conclude that $V(t) \in \mathcal{L}_{\infty}$. Signal chasing arguments can be used to prove that the remaining closed-loop signals are also bounded during the noncontact case provided that (56) is satisfied.

If the initial conditions for $V(0)$ are large enough that $\lambda\|y(t)\|^{2}>\varepsilon_{x}$, then the condition in (59) is sufficient. However, if the initial conditions for $V(0)$ are inside the region defined by $\varepsilon_{x}$, then $V(t)$ can grow larger until $\lambda\|y(t)\|^{2} \leq \varepsilon_{x}$. Therefore, further development is required to determine how large $V(t)$ can grow so that the sufficient condition in (56) can be satisfied. When $V(0)$ is inside the region defined by $\varepsilon_{x}$, then

$$
\|y(t)\| \leq \sqrt{\frac{\varepsilon_{x}}{\lambda}} .
$$

The expression in (60) can be used along with (24), (49), and (58) to conclude that

$$
\|\bar{z}(t)\| \leq \sqrt{5} \tanh ^{-1}\left(\sqrt{\frac{\varepsilon_{x}}{\lambda}}\right) .
$$

The inequality in (61) can be used along with (48) to rewrite the sufficient condition in (56) as

$$
k_{3} \alpha>\left(\sqrt{\frac{2\left(5 \lambda_{1}\left(\tanh ^{-1}\left(\sqrt{\frac{\varepsilon_{x}}{\lambda}}\right)\right)^{2}+\zeta_{\theta}\right)}{a_{1}}}+1\right)^{2} .
$$

Hence, the final sufficient condition for (56) is given by (39) where $V_{u} \in \mathbb{R}$ is defined as

$$
V_{u}=\max \left\{\lambda_{1}\|\bar{z}(0)\|^{2}+\zeta_{\theta}, 5 \lambda_{1}\left(\tanh ^{-1}\left(\sqrt{\frac{\varepsilon_{x}}{\lambda}}\right)\right)^{2}+\zeta_{\theta}\right\} .
$$

That is, provided that $k_{n 1}, \alpha, \alpha_{2}$, and $k_{3}$ are selected larger than known constants (that depends on the initial conditions of the states) according to (37), (38), and (39), all the states converge to an arbitrarily small neighborhood $\sqrt{\varepsilon_{x} / \lambda}$.

The previous development can be used to conclude that, for the noncontact case

$$
\|y(t)\| \rightarrow \sqrt{\frac{\varepsilon_{x}}{\lambda}}
$$


and hence

$$
\tanh \left(e_{f}\right), \tanh \left(\left\|e_{r}(t)\right\|\right) \rightarrow \sqrt{\frac{\varepsilon_{x}}{\lambda}} \quad \text { as } t \rightarrow \infty
$$

Based on (63)

$$
e_{f},\left\|e_{r}(t)\right\| \rightarrow \tanh ^{-1}\left(\sqrt{\frac{\varepsilon_{x}}{\lambda}}\right) \leq \varepsilon_{f}
$$

where $\varepsilon_{f} \in \mathbb{R}$ is a known positive constant as $t \rightarrow \infty$ for the noncontact case.

Further analysis is required to prove that the manipulator makes contact with the mass-spring system and to achieve the control objective. Contact between the manipulator and the mass-spring system occurs when $x_{r 1}(t) \geq x_{m}(t)$. Based on (64), a sufficient condition for contact can be developed as

$$
x_{r d 1} \geq x_{m}+\varepsilon_{f}
$$

After using (25), the sufficient condition in (65) can be expressed as

$$
Y_{d} \hat{\theta}_{d k}+k_{2} \tanh \left(e_{m}\right)-k_{1} k_{2} \cosh ^{2}\left(\varepsilon_{f}\right) \tanh \left(\varepsilon_{f}\right) \geq \varepsilon_{f} .
$$

By using (16) and (20) and performing some algebraic manipulations, the inequality in (66) can be expressed as

$k_{2} \tanh \left(e_{m}\right)-k_{1} k_{2} \cosh ^{2}\left(\varepsilon_{f}\right) \geq \varepsilon_{f}-x_{m d} \underline{\theta}_{d k}+\left(e_{m}+x_{0}\right) \bar{\theta}_{d k}$

where $\bar{\theta}_{d k}(t)$ and $\underline{\theta}_{d k}(t)$ are defined in (28). From Assumption $1, e_{m}(t)$ can be upper bounded as

$$
e_{m} \leq \bar{\varepsilon}_{m}
$$

where $\bar{\varepsilon}_{m} \in \mathbb{R}$ denotes a known positive constant. If $e_{m}(t) \leq 0$, then the sufficient condition in (67) may not be satisfied. The condition that $e_{m}(t) \leq 0$ will only occur if an impact collision that causes the mass to overshoot the desired position occurs. However, even if an impact occurs and the mass overshoots the desired position, the dynamics will force the mass position error to return to the initial condition. That is, $e_{m}(t) \rightarrow x_{m d}-x_{0}>$ $\underline{\varepsilon}_{m}$ where $\underline{\varepsilon}_{m} \in \mathbb{R}$ denotes a known positive constant. Based on (68) and the fact that $e_{m}(t)$ will eventually be lower bounded by $\underline{\varepsilon}_{m}$ in a noncontact condition, the inequality in (67) can be simplified as

$$
k_{2}\left(\tanh \left(\underline{\varepsilon}_{m}\right)-k_{1} \cosh ^{2}\left(\varepsilon_{f}\right)\right) \geq \varepsilon_{f}-x_{m d} \underline{\theta}_{d k}+\left(\bar{\varepsilon}_{m}+x_{0}\right) \bar{\theta}_{d k} .
$$

Based on (69), the control parameter $k_{2}$ can be selected according to the following sufficient condition to ensure that the robot and mass-spring system make contact:

$$
k_{2} \geq \frac{\varepsilon_{f}-x_{m d} \underline{\theta}_{d k}+\left(\bar{\varepsilon}_{m}+x_{0}\right) \bar{\theta}_{d k}}{\tanh \left(\underline{\varepsilon}_{m}\right)-k_{1} \cosh ^{2}\left(\varepsilon_{f}\right)}
$$

where $k_{1}$ is chosen as

$$
k_{1}<\frac{\tanh \left(\underline{\varepsilon}_{m}\right)}{\cosh ^{2}\left(\varepsilon_{f}\right)} .
$$

Case 2-Contact: For the case when the dynamic systems collide $(\Lambda=1)$ and the two dynamic systems become coupled ${ }^{1}$, (50) can be rewritten as

$$
\begin{aligned}
\dot{V} \leq & -k_{3} \tanh ^{2}\left(\left\|r_{r}\right\|\right)-\alpha_{1} k_{2} \underline{\zeta}_{K} \tanh ^{2}\left(e_{m}\right) \\
& -\alpha_{3} k_{2} \underline{\zeta}_{K} \tanh ^{2}\left(e_{f}\right)-3 \alpha_{2} \eta_{m}^{2}-\alpha \tanh ^{2}\left(\left\|e_{r}\right\|\right) \\
& -\left[\alpha\left\|e_{r}\right\|^{2}-\bar{\zeta}_{K} \mid \eta_{m}\left\|e_{r}\right\|\right] \\
& -\left[k_{n 1} \zeta_{1}^{2} \alpha_{2} \eta_{m}^{2}-\zeta_{1}\|z\| \eta_{m} \mid\right] \\
& -\left[\alpha\left\|e_{r}\right\|^{2}-2\left\|e_{r}\right\|\left\|r_{r}\right\|\right]
\end{aligned}
$$

where (11) was substituted for $K_{I}$ and (23) was substituted for $\chi\left(e_{m}, e_{f}, \eta_{m}, t\right)$. Completing the square on the three bracketed terms yields

$$
\begin{aligned}
\dot{V} \leq-\beta\|z\|^{2} & -\alpha \tanh ^{2}\left(\left\|e_{r}\right\|\right)-\left(\alpha_{2}-\frac{\bar{\zeta}_{K}^{2}}{4 \alpha}\right) \eta_{m}^{2} \\
& -\left(k_{3}-\frac{\left\|r_{r}\right\|^{2}}{\alpha \tanh ^{2}\left(\left\|r_{r}\right\|\right)}\right) \tanh ^{2}\left(\left\|r_{r}\right\|\right) .
\end{aligned}
$$

Because (47) is nonnegative, as long as (37), (38), and (39) are satisfied, (71) is negative semidefinite, and $r_{r}(t), \tilde{\theta}_{r}(t), \tilde{\theta}_{d k}(t)$, $e_{r}(t), e_{m}(t), e_{f}(t)$, and $\eta_{m}(t) \in \mathcal{L}_{\infty}$. Due to the fact that $e_{m}(t), e_{f}(t)$, and $\eta_{m}(t) \in \mathcal{L}_{\infty}$, the expression in (17) can be used to conclude that $\dot{e}_{m}(t) \in \mathcal{L}_{\infty}$ (and hence, $e_{m}(t)$ is UC). Due to the fact that $\dot{e}_{m}(t) \in \mathcal{L}_{\infty}, \dot{x}_{m}(t) \in \mathcal{L}_{\infty}$. Based on (4), $x_{m}(t) \in \mathcal{L}_{\infty}$. Previous facts can be used to prove that $x_{r d}(t) \in \mathcal{L}_{\infty}$, and since $e_{r}(t) \in \mathcal{L}_{\infty}$, then $x_{r}(t) \in \mathcal{L}_{\infty}$. Due to the fact that $e_{f}(t), e_{m}(t)$, and $\eta_{m}(t) \in \mathcal{L}_{\infty},(18)$ can be used to conclude that $\dot{e}_{f}(t) \in \mathcal{L}_{\infty}$. The expression in (19) can then be used to conclude that $\dot{\eta}_{m}(t) \in \mathcal{L}_{\infty}$ (and hence, $\eta_{m}(t)$ is UC). Based on (17) and the fact that $r_{r}(t)$ and $e_{r}(t) \in \mathcal{L}_{\infty}$, $\dot{e}_{r}(t) \in \mathcal{L}_{\infty}$. Furthermore, based on (28) and the fact that $x_{m}(t)$, $e_{m}(t), e_{f}(t), \eta_{m}(t)$, and $\dot{x}_{m}(t) \in \mathcal{L}_{\infty}$, the expression in (25) can be used to prove that $\dot{x}_{r d}(t) \in \mathcal{L}_{\infty}$. Based on the fact that $\dot{e}_{r}(t)$ and $\dot{x}_{r d}(t) \in \mathcal{L}_{\infty}$, the expression in (16) can be used to prove that $\dot{x}_{r}(t) \in \mathcal{L}_{\infty}$. Given that $x_{r}(t), \dot{x}_{r}(t), x_{m}(t), \dot{x}_{m}(t)$, $e_{f}(t)$, and $\eta_{m}(t) \in \mathcal{L}_{\infty}, Y_{r}(\cdot) \in \mathcal{L}_{\infty}$. The expression in (32) and (34) can then be used to prove that $F(t) \in \mathcal{L}_{\infty}$. The expression in (35) can be used to conclude that $\dot{r}_{r}(t) \in \mathcal{L}_{\infty}$ (and hence, $r_{r}(t)$ is UC). Since $e_{m}(t)$ and $r_{r}(t)$ are UC, which implies $\tanh \left(e_{m}(t)\right)$ and $\tanh \left(\left\|r_{r}(t)\right\|\right)$ are also UC, and because of the fact that $\tanh \left(e_{m}(t)\right), \tanh \left(\left\|r_{r}(t)\right\|\right)$, and $\eta_{m}(t)$ are square integrable, Barbalat's lemma can be used to conclude that $\tanh \left(e_{m}(t)\right), \tanh \left(\left\|r_{r}(t)\right\|\right)$, and $\left|\eta_{m}(t)\right| \rightarrow 0$ as $t \rightarrow \infty$, which also implies $\left|e_{m}(t)\right|$ and $\left\|r_{r}(t)\right\| \rightarrow 0$ as $t \rightarrow \infty$. Based on the fact that $\left\|r_{r}(t)\right\| \rightarrow 0$ as $t \rightarrow \infty$, standard linear analysis methods (see Lemma A.15 of [46]) can then be used to prove that $\left\|e_{r}(t)\right\| \rightarrow 0$ as $t \rightarrow \infty$.

\section{REFERENCES}

[1] A. Tornambe, "Modeling and control of impact in mechanical systems: Theory and experimental results," IEEE Trans. Autom. Control, vol. 44 no. 2, pp. 294-309, Feb. 1999.

[2] K. Youcef-Toumi and D. A. Guts, "Impact and force control," in Proc. IEEE Int. Conf. Robot. Autom., Scottsdale, AZ, 1989, vol. 1, pp. $410-416$.

\footnotetext{
${ }^{1}$ The dynamic systems can separate after impact; however, this case can still be analyzed under the nonimpact section of the stability analysis.
} 
[3] M. Indri and A. Tornambe, "Impact model and control of two multi-DOF cooperating manipulators," IEEE Trans. Autom. Control, vol. 44, no. 6, pp. 1297-1303, Jun. 1999.

[4] I. D. Walker, "The use of kinematic redundancy in reducing impact and contact effects in manipulation," in Proc. IEEE Int. Conf. Robot. Autom., Cincinnati, OH, 1990, vol. 1, pp. 434-439.

[5] M. W. Gertz, J. Kim, and P. K. Khosla, "Exploiting redundancy to reduce impact force," in Proc. IEEE/RSJ Int. Workshop IROS, Osaka, Japan, 1991, pp. 179-184.

[6] I. D. Walker, "Impact configurations and measures for kinematically redundant and multiple armed robot systems," IEEE Trans. Robot. Autom., vol. 10, pp. 346-351, 1994.

[7] D. Chiu and S. Lee, "Robust jump impact controller for manipulators," in Proc. IEEE/RSJ Int. Conf. Intell. Robots Syst., Pittsburgh, PA, 1995, vol. 1, pp. 299-304.

[8] A. Tornambe, "Global regulation of a planar robot arm striking a surface," IEEE Trans. Autom. Control, vol. 41, no. 10, pp. 1517-1521, Oct. 1996.

[9] P. R. Pagilla and B. Yu, "A stable transition controller for constrained robots," IEEE/ASME Trans. Mechatronics, vol. 6, no. 1, pp. 65-74, Mar. 2001.

[10] J. M. Hyde and M. R. Cutkosky, "Contact transition control: An experimental study," IEEE Control Syst. Mag., vol. 14, no. 1, pp. 25-31, 1994.

[11] P. Akella, V. Parra-Vega, S. Armoto, and K. Tanie, "Discontinuous model-based adaptive control for robots executing free and constrained tasks," in Proc. IEEE Int. Conf. Robot. Autom., San Diego, CA, 1994, vol. 4, pp. 3000-3007.

[12] T. Tarn, Y. Wu, N. Xi, and A. Isidori, "Force regulation and contact transition control," IEEE Control Syst. Mag., vol. 16, no. 1, pp. 32-40, Feb. 1996.

[13] Y. Wu, T. J. Tarn, N. Xi, and A. Isidori, "On robust impact control via positive acceleration feedback for robot manipulators," in Proc. IEEE Int. Conf. Robot. Autom., Albuquerque, NM, 1996, pp. $1891-1896$

[14] E. Lee, "Force and impact control for robot manipulators using time delay," in Proc. IEEE Int. Symp. Ind. Electron., 1999, pp. 151-156.

[15] E. Lee et al., "Bang-bang impact control using hybrid impedance timedelay control," IEEE/ASME Trans. Mechatronics, vol. 8, no. 2, pp. 272-277, Jun. 2003.

[16] B. J. Nelson, J. D. Morrow, and P. K. Khosla, "Fast stable contact transitions with a stiff manipulator using force and vision feedback," in Proc. IEEE/RSJ Int. Conf. Intell. Robots Syst., Pittsburgh, PA, 1995, vol. 2, pp. 90-95.

[17] Y. Zhou, B. J. Nelson, and B. Vikramaditya, "Fusing force and vision feedback for micromanipulation," in Proc. IEEE Int. Conf. Robot. Autom., Leuven, Belgium, 1998, pp. 1220-1225.

[18] G. Yang and B. J. Nelson, "Micromanipulation contact transition control by selective focusing and microforce control," in Proc. IEEE Int. Conf. Robot. Autom., Taipei, Taiwan, 2003, pp. 3200-3206.

[19] M. Shibata and T. Natori, "Impact force reduction for biped robot based on decoupling COG control scheme," in Proc. IEEE Int. Workshop Advanced Motion Control, Nagoya, Japan, 2000, pp. 612-617.

[20] E. Ohashi and K. Ohnishi, "Motion control in the support phase for a one-legged hopping robot," in Proc. IEEE Int. Workshop Advanced Motion Control, Kawasaki, Japan, 2004, pp. 259-262.

[21] Y. Sato, E. Ohashi, and K. Ohnishi, "Impact force reduction for hopping robot," in Proc. Conf. IEEE Ind. Electron. Soc., 2005, pp. 1821-1826.

[22] R. V. Dubey, T. F. Chan, and S. E. Everett, "Variable damping impedance control of a bilateral telerobotic system," IEEE Control Syst. Mag., vol. 17, no. 1, pp. 37-45, Feb. 1997.

[23] Y. Yamada, Y. Hirasawa, S. Huang, Y. Umetani, and K. Suita, "Human-robot contact in the safeguarding space," IEEE/ASME Trans. Mechatronics, vol. 2, no. 4, pp. 230-236, Dec. 1997.

[24] Z. Li, A. Ming, N. Xi, Z. Xie, J. Gu, and M. Shimojo, "Collision-tolerant control for hybrid joint based arm of nonholonomic mobile manipulator in human-robot symbiotic environments," in Proc. IEEE Int. Conf. Robot. Autom., Barcelona, Spain, 2005, pp. 4037-4043.

[25] P. Huang, Y. Xu, and B. Liang, "Contact and impact dynamics of space manipulator and free-flying target," in Proc. IEEE/RSJ Int. Conf. Intell. Robots Syst., 2005, pp. 1181-1186.

[26] M. Indri and A. Tornambe, "Control of under-actuated mechanical systems subject to smooth impacts," in Proc. IEEE Conf. Decision Control, Atlantis, Bahamas, 2004, pp. 1228-1233.

[27] K. Dupree, W. E. Dixon, G. Hu, and C. Liang, "Lyapunov-based control of a robot and mass-spring system undergoing an impact collision," in Proc. IEEE Amer. Control Conf., Minneapolis, MN, 2006, pp. $3241-3246$
[28] K. Dupree, C. Liang, G. Hu, and W. E. Dixon, "Global adaptive Lyapunov-based control of a robot and mass-spring system undergoing an impact-collision," in Proc. IEEE Conf. Decision Control, San Deigo, CA, 2006, pp. 2039-2044.

[29] K. Dupree, C. Liang, G. Hu, and W. E. Dixon, "Adaptive Lyapunovbased control of a robot and massspring system undergoing an impact collision," IEEE Trans. Syst., Man, Cybern. B, Cybern., vol. 38, no. 4, pp. 1050-1061, Aug. 2006.

[30] G. Hu, W. E. Dixon, and C. Makkar, "Energy-based nonlinear control of under-actuated Euler-Lagrange systems subject to impacts," in Proc. IEEE Conf. Decision Control, Seville, Spain, 2005, pp. 6859-6864.

[31] B. Brogliato, Nonsmooth Impact Mechanics. London, U.K.: Springer-Verlag, 1996.

[32] B. Brogliato, S. I. Niculescu, and P. Orhant, "On the control of finite-dimentional mechanical systems with unilateral constraints," IEEE Trans. Autom. Control, vol. 42, no. 2, pp. 200-215, Feb. 1997.

[33] B. Brogliato and P. Orhant, "Contact stability analysis of a one degree-of-freedom robot," Dyn. Control, vol. 8, no. 1, pp. 37-53, Jan. 1998.

[34] B. Brogliato and A. Z. Rio, "On the control of complementary-slackness juggling mechanical systems," IEEE Trans. Autom. Control, vol. 45, no. 2, pp. 235-246, Feb. 2000.

[35] P. R. Pagilla and M. Tomizuka, "Contact transition control of nonlinear mechanical systems subject to a unilateral constraint," Trans. ASME, J. Dyn. Syst. Meas. Control, vol. 119, no. 4, pp. 149-159, 1997.

[36] P. R. Pagilla, "Control of contact problem in constrained Euler-Lagrange systems," IEEE Trans. Autom. Control, vol. 46, no. 10, pp. 1595-1599, Oct. 2001

[37] P. R. Pagilla and B. Yu, "Mechatronic design and control of a robot system interacting with an external environment," Mechatronics, vol. 12, no. 6, pp. 791-811, Jul. 2002.

[38] P. R. Pagilla and B. Yu, "An experimental study of planar impact of a robot manipulator," IEEE/ASME Trans. Mechatronics, vol. 9, no. 1, pp. 123-128, Mar. 2004.

[39] X. Cyril, G. J. Jarr, and A. K. Misra, "The effect of payload impact on the dynamics of a space robot," in Proc. IEEE/RSJ Int. Conf. Intell. Robots Syst., Yokohama, Japan, 1993, vol. 3, pp. 2070-2075.

[40] D. Materassi, M. Basso, and R. Genesio, "A model for impact dynamics and its application to frequency analysis of tapping-mode atomic force microscopes," in Proc. IEEE Conf. Decision Control, 2004, vol. 6, pp. $6218-6223$.

[41] L. Menini and A. Tornambe, "Asymptotic tracking of periodic trajectories for a simple mechanical system subject to nonsmooth impacts," IEEE Trans. Autom. Control, vol. 46, no. 7, pp. 1122-1126, Jul. 2001.

[42] P. Sekhavat, Q. Wu, and N. Sepehri, "Impact control in hydraulic actuators with friction: theory and experiments," in Proc. Amer. Control Conf., Boston, MA, 2004, pp. 4432-4437.

[43] R. Volpe and P. Khosla, "A theoretical and experimental investigation of impact control for manipulators," Int. J. Robot. Res., vol. 12, no. 4, pp. 670-683, 1994.

[44] K. Yoshida, C. Mavroidis, and S. Dubowsky, "Impact dynamic of space long reach manipulators," in Proc. IEEE Int. Conf. Robot. Autom., Minneapolis, MN, 1996, vol. 3, pp. 1909-1916.

[45] W. E. Dixon, M. S. de Queiroz, D. M. Dawson, and F. Zhang, "Tracking control of robot manipulators with bounded torque inputs," Robotica, vol. 17, no. 2, pp. 121-129, Mar. 1999.

[46] W. E. Dixon, A. Behal, D. M. Dawson, and S. Nagarkatti, Nonlinear Control of Engineering Systems: A Lyapunov-Based Approach. Boston, MA: Birkhäuser, 2003.

[47] W. E. Dixon, E. Zergeroglu, D. M. Dawson, and M. W. Hannan, "Global adaptive partial state feedback tracking control of rigid-link flexible-joint robots," Robotica, vol. 18, no. 3, pp. 325-336, May 2000.

[48] Z. Cai, M. S. de Queiroz, and D. M. Dawson, "A sufficiently smooth projection operator," IEEE Trans. Autom. Control, vol. 51, no. 1, pp. 135-139, 2006.

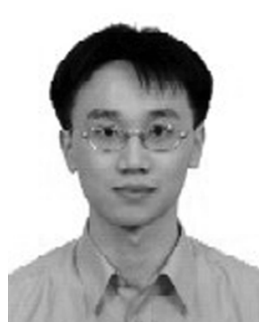

Chien-Hao Liang (S'06) received the B.S. degree in ocean engineering from National Taiwan University, Taipei, Taiwan, in 2001 and the M.S. degree in mechanical and aerospace engineering from the University of Florida, Gainesville, in 2007.

He is currently with the Department of Mechanical and Aerospace Engineering, University of Florida. His research interest is in impact control of robot manipulators. 


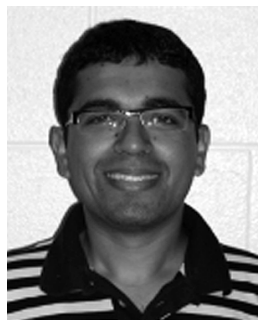

Shubhendu Bhasin (S'08) received the B.E. degree in manufacturing processes and automation engineering from the Netaji Subhas Institute of Technology (formerly, Delhi Institute of Technology), New Delhi, India, in 2004. He is currently working toward the Ph.D. degree in the Department of Mechanical and Aerospace Engineering, University of Florida, Gainesville.

He was an Embedded Systems Engineer with Tata Elxsi Ltd., India, and Conexant Systems Ltd., India. His research interests include nonlinear adaptive and neural-network control of mechanical systems undergoing impact.

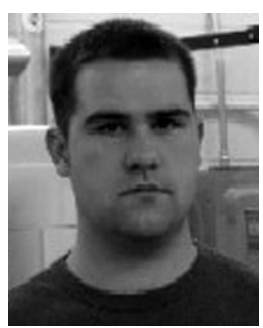

Keith Dupree (S'06) received the B.Sc. degree in aerospace engineering with a minor in physics from the University of Florida, Gainesville, in 2005, where he is currently working toward the M.S. degree in the Department of Electrical and Computer Engineering and the Ph.D. degree in the Department of Mechanical and Aerospace Engineering, as a member of the Nonlinear Controls and Robotics Group, both under the advisement of Dr. Warren Dixon.

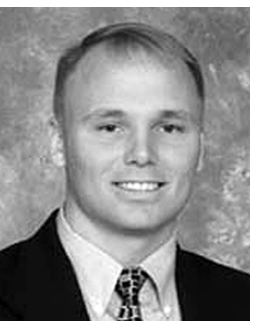

Warren E. Dixon (S'94-M'00-SM'05) received the Ph.D. degree from the Department of Electrical and Computer Engineering, Clemson University, Clemson, SC, in 2000.

After completing his doctoral studies, he was selected as a Eugene P. Wigner Fellow with the Oak Ridge National Laboratory. Since 2004, he has been with the faculty of the Department of Mechanical and Aerospace Engineering, University of Florida, Gainesville. He has published two books and over 150 journal and conference papers on the development and application of Lyapunov-based control methods.

Dr. Dixon's efforts in this area have been acknowledged through awards such as a National Science Foundation CAREER Award and the IEEE Robotics and Automation Society Early Academic Career Award. He is a member of numerous conference program committees, technical committees, organizing committees, and conference editorial boards. He is an appointed member of the IEEE Control Systems Society Board of Governors and is currently an Associate Editor for the IEEE TRANSACTIONS ON SYSTEMS, MAN, AND CYBERNETICS-PART B: CYBERNETICS, Automatica, International Journal of Robust and Nonlinear Control, and Journal of Robotics. 